Dhaka Univ. J. Biol. Sci. 26(2): 117-123, 2017 (July)

\title{
TRANSLATION AND ADAPTATION OF PARENTAL-CHILD ATTACHMENT SCALE IN BANGLA
}

\author{
Abu Zafar Md Riaz Uddin, Umme KaWSER*, Rajon Das Jony \\ AND AKIB Ul HuQue \\ Department of Educational and Counselling Psychology, University of Dhaka, \\ Dhaka-1000, Bangladesh
}

Key words: Adolescent, Parental-child, Attachment

\begin{abstract}
Hudson's parental-child attachment scale (PCAS) is a popular instrument to assess the quality of parental child attachment - the emotional bond that influences the social and emotional development of a child. The present study adapted this scale for using with Bangla speaking parents and their children. This study was conducted with 100 children and their parents (either father or mother). The internal consistency of the parental-child attachment scale was computed by Cronbach's alpha which was 0.888 for youth items and 0.816 for parent items. The split-half reliability co-efficient of the scale was 0.787 for youth items and 0.779 for parent items. So the preliminary reliability and content validity of the scale has been established.
\end{abstract}

\section{Introduction}

Parental-child attachment is an important predictor that gives insight into family bonding. Studies have demonstrated the importance of parental child attachment for adjustment, indiscipline style during childhood and adolescence. There are many different attachments formed over the course of the life span, the attachment between parents and child is among the most important ${ }^{(1)}$. Not surprisingly, studies of child development have devoted considerable attention to the parental-child attachment, in order to understand how it develops and functions over the life span. Research has found that a loving, responsive, and helpful parent who is always available for their child serves the function of bonding the child to them and contributes to the reciprocal dynamics of that bonding ${ }^{(2)}$. However, there is not sufficient evidence to declare its efficacy.

The study of secure parental-child attachment in child development has received ever increasing attention in the last few decades with special focus towards the cognitive, social and emotional development ${ }^{(3)}$. Efforts to explore the effects of home environment on child development have yielded consistent findings of association between parenting practices and social, emotional and cognitive development ${ }^{(4)}$.

*Author for correspondence: <kawserumme@du.ac.bd>. 
Indeed available data show that vigorous parental-child attachments have been associated with positive outcome for adolescents protecting them from psychological, emotional and behavioral problems ${ }^{(5)}$. While unhealthy interaction of parents with children may lead towards many emotional, behavioral and cognitive problems in children ${ }^{(6-8)}$. Concept of parental-child attachment has defined as an interaction of the parents with their children consists of a combination of feelings, perceptions, expressions, behaviors, and expectations that relate parents to their children. Cordial parental-child attachment includes parental warmth, love, affection, support and parental reward. Negative parental-child attachment includes parental rejection, neglecting, punishment and indifferent behavior towards the child. Though the attachment with parents changes during adolescence, yet the quality of parental-child attachment is still an important determinant of healthy adjustment of adolescents. Transition to adolescence becomes healthy and easy in children who enjoy warm and positive relations with parents. Therefore parents need to recognize the continued importance of positive and loving attachments with their growing children during this critical developmental period ${ }^{(9)}$.

During the past several decades, cross-cultural assessment by using the standardized tests has become a sensitive issue ${ }^{(10)}$. Equivalence of psychological measures across cultural groups can be ensured by establishing the external validity in addition to internal validity and by cross validation of the measures across cultures ${ }^{(11)}$. For cross cultural testing, test translation and test adaptation were identified as two main issues by Butcher and Garcia ${ }^{(12)}$. Fouad ${ }^{(13)}$ and Geisinger ${ }^{(14)}$ stated that instrument must be validly adapted and conceptual and linguistic equivalence of the items must be maintained during the process of translation. With increasing concern in parent-child attachment in developmental studies, it is important to establish the cross validity of the parental-child attachment scales across different cultures and languages.

So the primary aim of the study was the translation and adaptation of the of the parental-child attachment scale into Bangla, the national language of Bangladesh. There is a dire need to address youth adjustment in light of parent child attachments for which currently no scales are available. Additionally, translation and adaptation of parentalchild attachments scale is necessary for cross cultural comparison of parenting practices. So the study primarily is intended to translate and cross validate the parental-child attachment scale into Bangla, to be available for Bangladeshi researchers to address youth adjustment problems in light of parenting and to facilitate cross cultural evaluation of parenting practices.

The studies further intended to establish the reliability and validity the Bangla version by analyzing the psychometric properties of the scale. Problems faced by Bangladeshi youth have never been paid due consideration and addressed properly. Therefore it is important to understand the impact of parental-child relations on 
adolescent development in Bangladeshi population. In this situation, if these scales are adapted in Bangladesh context, it would help to measure parental child attachment of Bangladeshis. This study will also be helpful for better understanding of parental-child attachment and the importance of positive parenting.

Main objectives of the study were as follows.

1. To adapt the parental-child attachment scale into Bangla language.

2. To establish the psychometric properties of parental-child attachment scale in Bangla version.

\section{Material and Methods}

In this study, 100 participants were chosen covering age range from 11 to 15 years. Among them 52 were girls and 48 were boys and also 100 were their parents (either father or mother). Total 200 participants were taken to conduct the adaptation process of this scale. The participants were chosen from three different schools in Dhaka city following convenient sampling technique.

For data collection two materials were used: (i) Demographic information including age, sex, class of the student and (ii) Bangla version of parental-child attachment scale.

Parental-child attachment scale (PCAS) was adapted from Hudson 1982(15). This scale has 22 items (11 child items and 11 parent items) that measure the degree of warmth and lack of hostility in the parent child attachment. This scale has two separate parts which are youth items and parent items. Internal consistency of youth items is 0.87 and parent items is 81 . Parent and youth items are scored separately.

The translation and adaptation of parental-child attachment scale involved several multi-stage procedures which are being described step by step.

At first, the scale was translated into Bangla language by two independent translators whose original mother tongue was Bangla. They were required to translate the scale from English into Bangla by following some guidelines provided by the author.

- To focus on the content similarity between Bangla translation and original English version.

- To maintain the difficulty level (low) of the original scale.

- To translate the items without omission and elimination of items.

- To translate items in language which readily understandable in Bangladeshi culture.

After that, the expert panel comprising the principal researcher and the supervisor compared the two different translations and assessed their semantic, idiomatic, conceptual, linguistic and contextual differences. After that they synthesized a single translated version.

Then the synthesized version was evaluated by three experts who had the knowledge of what the instrument assessed. These experts evaluated note worthy features, such as 
the structure, layout, instrument instructions, and both the scope and adequacy of expressions contained in the items.

In third stage, the Bangla translated version was given to two experts of the Department of English of University of Dhaka for back translation which was a process of validity inspection to check that the translated version was reflecting the same item content as the original versions. They had not seen the original parental-child attachment scale before.

Finally, after synthesizing all the documents by expert review committee, the final Bangla version of parental-child attachment scale was prepared and administered to 200 participants falling in age range of 11 - 15 years and 100 of their parents (either father or mother). Data were collected purposefully and participants who were interested in participating were recruited. They were given both verbal and written instruction about what to do and how to fill up the questionnaire.

\section{Results and Discussion}

Descriptive statistics of the study shows that the mean score for youth items was 35.28 with a standard deviation of 6.08 . The lowest possible score is 20 and the highest possible score is 43 . For the parent items mean score were 31.03 with standard deviation of 5.25. The lowest possible score is 16 and the highest possible score is 42 .

Table 1. Mean $(X)$ and standard deviation (sd) of parental-child attachment (youth and parent items) $(n=200)$.

\begin{tabular}{lcccc}
\hline Scales & Min. & Max. & Mean & Sd \\
\hline Parental-child attachment & & & & \\
Youth items & 20.00 & 43.00 & 35.2800 & 6.08040 \\
Parent items & 16.00 & 42.00 & 31.0300 & 5.25982 \\
\hline
\end{tabular}

Table 2. Reliability coefficient of Bangla version of the parental-child attachment scale.

\begin{tabular}{lcc}
\hline Parental-child & Cronbach's alpha & Split-half \\
Attachment scale & reliability coefficient $(\mathrm{n}=100)$ & reliability \\
Youth items & $0.888^{*}$ & $0.787^{*}$ \\
Parent items & $0.816^{*}$ & $0.779^{*}$ \\
\hline
\end{tabular}

*Correlation is significant at the 0.05 level (2-tailed).

The internal consistency of the PCAS was computed by Cronbach's alpha which was 0.888 for youth items and 0.816 for parent items. The split-half reliability coefficient of the PCAS was 0.787 for youth items and 0.779 for parent items. 
Item analysis of a Likert-type scale is done by calculating the correlation between individual item's score with the total scale score. For youth and parent item, all 11 items were thoroughly analyzed and corrected item-total correlation coefficients were determined and shown in Tables 3 and 4 .

Table 3. Youth item-total statistic.

\begin{tabular}{lccc}
\hline $\begin{array}{l}\text { Youth } \\
\text { items }\end{array}$ & $\begin{array}{c}\text { Scale mean if item } \\
\text { deleted }\end{array}$ & $\begin{array}{c}\text { Scale variance if item } \\
\text { deleted }\end{array}$ & $\begin{array}{c}\text { Cronbach's alpha if } \\
\text { item deleted }\end{array}$ \\
\hline 1 & 31.8200 & 29.785 & 0.867 \\
2 & 31.8700 & 29.124 & 0.866 \\
3 & 32.1800 & 32.553 & 0.893 \\
4 & 32.3400 & 31.843 & 0.891 \\
5 & 32.0800 & 30.680 & 0.874 \\
6 & 31.9600 & 30.261 & 0.873 \\
7 & 32.2300 & 31.290 & 0.879 \\
8 & 32.1500 & 30.614 & 0.875 \\
9 & 32.2000 & 31.838 & 0.884 \\
10 & 31.9000 & 31.081 & 0.877 \\
11 & 32.0700 & 30.793 & 0.878 \\
\hline
\end{tabular}

Table 4. Parent item-total statistics.

\begin{tabular}{lccc}
\hline $\begin{array}{l}\text { Parent } \\
\text { items }\end{array}$ & $\begin{array}{c}\text { Scale mean if item } \\
\text { deleted }\end{array}$ & $\begin{array}{c}\text { Scale variance if item } \\
\text { deleted }\end{array}$ & $\begin{array}{c}\text { Cronbach's alpha if } \\
\text { item deleted }\end{array}$ \\
\hline 1 & 27.8100 & 23.166 & 0.796 \\
2 & 27.9300 & 22.369 & 0.792 \\
3 & 28.5900 & 22.608 & 0.796 \\
4 & 28.7000 & 22.899 & 0.797 \\
5 & 27.8900 & 25.210 & 0.816 \\
6 & 28.3500 & 22.432 & 0.790 \\
7 & 28.2300 & 24.583 & 0.806 \\
8 & 28.3900 & 22.968 & 0.803 \\
9 & 28.1400 & 22.081 & 0.797 \\
10 & 28.1500 & 24.553 & 0.811 \\
11 & 28.1200 & 23.278 & 0.802 \\
\hline
\end{tabular}


Content validity of the scale was assured by checking items during construction of the test.

Although the adaptation of the parent-child attachment scale into Bangla was considered appropriate; the study had a number of recommendations. Qualitative methodology or factor analysis of data should be undertaken in future to provide sufficient proof of appropriateness in Bangladeshi culture and population. In the present study, data were taken only from different parts of Dhaka city. A larger and more comprehensive sample of all over Bangladesh would be taken for enhanced results. So, validation studies over the general population should be conducted by taking a countrywide sampling and compared to the validation with this participant.

\section{References}

1. Steinberg L 2001. We know some things. Parent-adolescent relationship in retrospect and prospect. J. Res. Adolescence 11(1): 1-19.

2. Bowlby J 1988. A secure base: Parent-child attachment and healthy human development. New York: Basic Books.

3. Erickson MF, LA Sroufe and B Egeland 1985. The relationship between quality of attachment and behavior problems in preschool in a high-risk sample. Monographs of the Society for Research in Child Development 50(1): 147-166.

4. El-Sheikh M and JA Buckhalt 2003. Parental problem drinking and children's adjustment: Attachment and family functioning as moderators and mediators of risk. J. Family Psychol. 17(4): 510-520.

5. Brody GH, VM Murry, L McNair, Y Chen, FX Gibbons and M Gerrard 2005. Linking changes in parenting to parent-child relationship quality and youth self-control: The strong African American families program. J. Res. Adolescence 15(1): 47-69.

6. Fatima S and H Sheikh 2009. Aggression in adolescents as a function of parent-child relationship. Pakistan J. Psychol. 40(2): 3-14.

7. Harrist AW and RC Ainslie 1998. Marital discord and child behavior problems: Parent-child relationship quality and child interpersonal awareness as mediators. Journal of Family Issues 19: 140-163.

8. McNeal Jr. RB 2001. Differential effects of parental involvement on cognitive and behavioral outcomes by socioeconomic status. Journal of Socio-Economics 30: 171-179.

9. Brittain C and DE Hunt (Eds.) 2003. American Human's Helping in Child Protective Services: A Competency-based Casework Handbook. New York: Oxford University Press.

10. Chang CY 2001. Cross-cultural assessment: A call for test adaptation. Association for Assessment in Counseling. 
11. Sue S 1999. Science, ethnicity and bias: Where have we gone wrong? American Psychologist 54(12): 1070-1077.

12. Butcher JN and RE Garcia 1978. Cross-national application of psychological tests. The Personnel and Guidance Journal 56: 472-475.

13. Fouad NA 1993. Cross-cultural vocational assessment. The Career Development Quarterly 42: 4-13.

14. Geisinger KF 1994. Cross-cultural normative assessment: Translation and adaptation issues influencing the normative interpretation of assessment instruments. Psychological Assessment 6: 304-312.

15. Hudson W 1982. The Clinical Measurement Package: A Field Manual. Homewood, IL: Dorsey Press.

(Manuscript received on 11 November, 2016; revised on 21 May, 2017) 